\title{
On the Computer Modeling for Musical Thinking
}

\author{
Sergei Filatov-Beckmann \\ Russian State Special Academy of Arts \\ Tchaikovsky Moscow State Conservatory \\ Moscow, Russia \\ E-mail: mserg1958@mail.ru
}

\begin{abstract}
Methods of mathematical modeling for musical thinking are one of the current problems of modern musical science. A number of works by Professor S. Skrebkov, to whom the problem statement belongs, are under analysis. The reasons for exceptional complexity of the problem are considered, and approaches are formulated to solve it from today's standpoint.
\end{abstract}

Keywords-computer modeling; "soft" modeling; artistic integrity; self-organizatio; non-linear system; signal; phase reconstruction

\section{INTRODUCTION}

Of recent trends, there has been an increasing role of computers within the humanitarian field of scientific endeavor. Computing devices in the recent past and personal computers (laptop, tablet, etc.) at present serve as a basic tool and sometimes as a co-author of many important results.

The statement of the first approaches the problem of machine (computer) modeling in the field which is far away from technical sciences that belongs to early 60 s of the last century and is largely associated with the name of the outstanding Russian musical thinker S. Skrebkov (19051967). His heritage is as large and multifaceted as any heritage of a scientist who formulated the questions for the answers to which are to be found by future generations. The present work addresses some of these issues.

It is worth recalling that in those years which are already distant from the present day, the mathematical modeling made only the first steps based on electron-tube computer engineering. The digital systems were mainly cyclopean structures, requiring constant control (as we would say today -monitoring). Magnetic memory was estimated by megabytes (at best) and the speed of elementary operations perhaps thousands per second. It was a long way to computing devices, even on the basis of semiconductor technology, not even mentioning modern parameters of memory media and performance of processors.

However, from small beginnings come great things. Not everyone can see germs of the future in routine of the present, but these germs always exist. Gradually, the accumulated amount will result in a new quality, and the problems which are almost unrealizable for computer engineering of the recent past will be fully solved on the basis of today's personal computer.
This situation could be illustrated by a significant number of examples from various applicable areas. However, in our opinion, a fairly general view of the computer simulation problem would be more convincing, which allows to offer a classification of tasks corresponding to a given field of knowledge [6].

\section{COMPUTER MODELING: ClASSIFICATION OF TASKS}

Thus, we can formulate at least four complex levels of problems that are encountered by the researcher. The first level involves an analytical solution with the help of "pencil and paper"; a significant body of such problems were implemented in the second half of the twentieth century (quantum theory, some issues of hydrodynamics, plasma theory, and so on). The second level includes the problems and the solution of which is possible only on the basis of computer engineering (designing reactors, climate forecast, earthquake prediction, issues of aviation and space technology). The third level includes the problems and the solution of which requires an extremely powerful computer resource capacity (large-scale economic and environmental forecasts, evolution of the sun, stars and nebulae, fundamental questions of the origin of life).

\section{ABout Computer Modeling In Musical ScIENCE}

The three levels of problems have in common the point that the original equations for their solution are known. However, there is a fourth level, and it includes modeling in the field of humanitarian studies (history, pedagogy and psychology). For the recent 15-20 years, this trend was called "soft modeling". Computer modeling techniques take the first steps into the scientific study of music.

The impossibility of resolution of a scientific problem is dualistic: it is a question of either principle or technical incomprehension. In the second case, the problem is likely to be solved (although it may have to wait for the desired solution for a long time). In the first case, the "barrier" can be inaccessible.

The above can be applied to "soft modeling" problems. Let's try to understand the nature of the fundamental difficulties. Let's refer to one of the last works of prof. S. Skrebkov "On the methodological principles of musical thinking modeling". The article prepared at the turn of 196566 was published only almost forty years later, long after the 
death of S. Skrebkov, in the collection dedicated to the 100th anniversary of the birth of the author [5].

One of the issues raised by S. Skrebkov in this article is as follows: a musical masterpiece cannot contain the number of sounds below a certain critical value; this value, according to the author, should be at least 200. S. Skrebkov writes: "We allow ourselves to make the following suggestion. It is possible that qualitative transitions from sound to sound in tune and from one its section to another are committed, if expressed by mathematical language, under the laws of nonlinear continuous functions ... [5, p. 241]. The author hypothesizes that the number of sounds, no less than critical one, is necessary to ensure that "... a complex function in all its artistic integrity... could form" in the mind of a listener [5, p.241], and that function (or, rather, the operator) should be of essentially nonlinear character.

It is difficult to overestimate quite modern sounding of the cited text; one gets the impression that the text was created today, not half a century ago, and focuses on the urgent problems of our time (such time invariance is an inherent property of truly outstanding ideas).

Further, exploring the text of the article, let's cite the following statement of the author: "In its individual sections or parts the masterpiece (of art - SWF) can be a little bit similar to other works, but on the whole, as a single "entity", it is always uniquely peculiar and thus incredibly complicated" [5, p.241]. When reasoning in terms of modern synergy, the contours of complex dynamic (perhaps even cybernetic) system are distinctly seen in this characteristic of the (musical) masterpiece, certainly possessing the phenomenon of independent behavior. It is able to show certain features of self-organization.

Let's address another work of S. Skrebkov, published in the book cited and entitled "Why the possibilities of classical forms are inexhaustible". In this article, small by volume, but very deep by content, the author formulates the following thought: "Musical logic, by virtue of extreme flexibility, dialectical diversity and interesting content can serve to creative practice of different composers of different eras. But musical logic is not omnivorous, it has an accurate measure of its principles, although encompasses an endless range of possibilities: its measure is orientation on a listener, fundamental artistic democracy in setting an aesthetic problem of impact on the musical consciousness of people [5, p. 257]

Thus, a piece of music (and especially an outstanding one) can be represented as a dynamic system with the following features: such system is nonlinear and is characterized by incredible complexity; to study this system, a measure of a construct should be introduced that can influence musical consciousness.

It is well known that the theory of measure is one of the fundamental issues of functional analysis - one of the foundations of modern mathematics. One might say that the measure as a kind of general characteristic is designed to reflect the most important features of certain mathematical structures; as an elementary example, the measure can be considered as the area of a rectangle (open or closed). As you know, a fundamental contribution to the construction of the theory of measure was made by the outstanding French mathematician Lebesgue, who extended this concept on a wider class of mathematical objects $[1,2]$.

The brief analysis of two articles of S. Skrebkov allows us to find the answer to the question about the reasons for the principal difficulties in the field of "soft modeling" stated above. Let's begin with the analysis of the measure introduced for the study of the musical composition. The fact of focusing on the listener and impact on his musical consciousness can be formally interpreted in terms of wellknown modern nonlinear dynamics: the signal (i.e., focus on the listener) and the response of the system (i.e., impact on the musical consciousness). The signal may be represented as a superposition of non-linear functions (for example, on the basis of Fourier series theory). However, the principal difficulty lays in the fact that for more or less acceptable description of the response of the system, sufficiently detailed understanding is required of the mechanisms of perception of audio information by the human brain. The situation is complicated by the fact that, according to $\mathrm{R}$. Penrose, the basis of brain activity is a certain not computable process [7].

The idea of R. Penrose can be commented as follows. Suppose that there is some simple signal $x(\mathrm{t})$, which is perceived by some system. This perception creates a system response $h(\tau-\mathrm{t})$, where $\mathrm{t}$ and $\tau$ - some time stations, with $\tau$ more than $t$ (i.e., the effect cannot occur before the cause). Suppose that there is a multiple generation of $x(\mathrm{t})$ signal; then the integral sum of the products (or the so-called convolution) will identify system response to the generated signals.

The complexity of the problem lies mainly in the construction of the function $h$ of system response: it requires the creation of a computer model of the perception of the field of musical information by human brain.

\section{FIRST Results OF COMPUTER-Musical MODELING}

Could this problem be solved, at least in principle? Today's learning level does not allow any comprehensive answer to this question. However, there is an intermediate institution (in a manner, local) solution between the problem posed (simulation of musical thinking) and its final resolution. On the search path of the intermediate solutions, certain successes can be achieved.

Thus, computer methods for studying elementary music signals can detect features not distinguished by a human ear. Here is an example of the so-called phase reconstruction of two noise signals-white (WN) and pink (PN) noise (phase reconstruction is constructed by arranging linearly independent values of the same signal on the axes of the plane frame) "Fig. 1": 

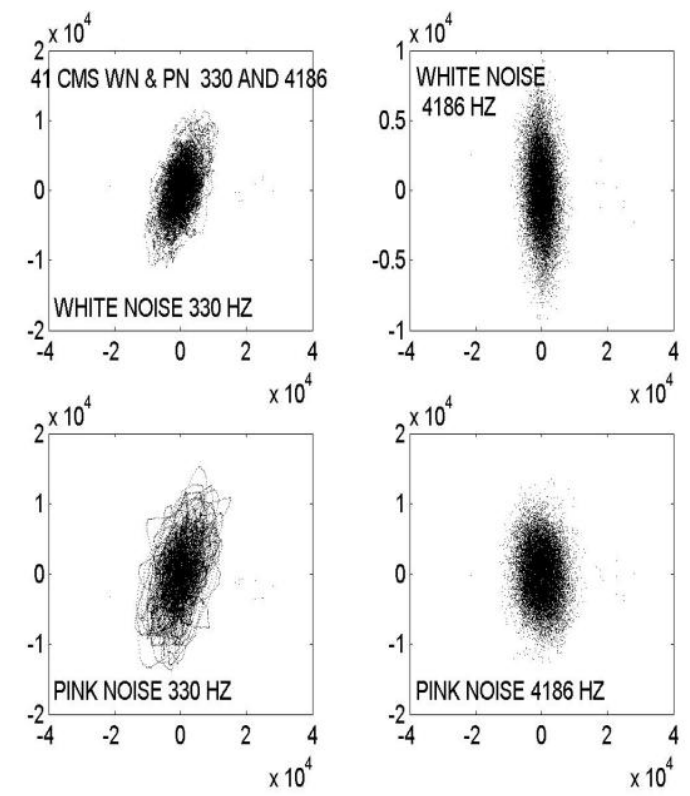

Fig. 1. Phase Reconstructions for Noise Signals

The distinguished signals are obtained in the mode of the so-called narrowband implementation: the reference frequencies of $330 \mathrm{~Hz}$ and $4186 \mathrm{~Hz}$ are central values of the frequency bands of 0.1 octave (or 120 cents) width.

The figure shows that even the elementary signals generate phase images differing by rather complicated topology. Such images can be constructed and studied on the basis of the author's musical and statistical model, which operates by both purely statistical characteristics and variables belonging to the modern nonlinear dynamics [3].

Without discussing in detail the properties of the resulting images, we may note that only they have inherent fractal features generated by the so-called quantization noise occurring in the synthesis of "digitized" music signal.

Much more complicated phase images generate records of performance of musical instruments.

"Fig. 2" shows the reconstruction of a guitar chord:

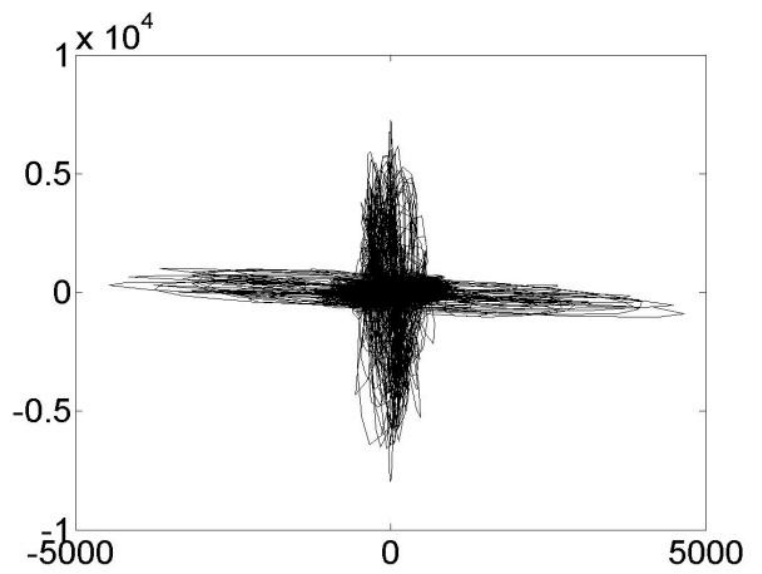

Fig. 2. Phase Reconstruction of Guitar Chord
The fact which seems to us to be worthy is that, in contrast to the previous figure, the line spaces connecting the imaging points have a direction perpendicular to each other; the image as a whole resembles the so-called "strange attractor" [3].

The complexity of the topology of the reconstructions of considered types of signals indicates a high level of complexity of the music signals themselves. However, "Fig. 2 " is generated by an actual performance and, therefore, bears a reflection of the mentality of the performing musician.

\section{Mathematical Music As the SAMPLE of DETERMINED COMPUTER MUSIC}

Above we have briefly discussed the concept of mathematical music, as well as methods for its synthesis. Let's contemplate this issue more elaborately.

There is a significant role of computer music in the extensive range of modern electro-acoustic music. This music is generated purely by computer means. Thus, the composer, who uses a synthesizer, creates a symphonic work, taking advantage of vast electronic timbre banks of sounds of musical instruments. The piece of music can meet both classical musical form and any of the areas of modern compositions; in this case, the computer plays the role of a "fixator" of creative musical thought.

However, there is another approach: the computer becomes a musical co-author of the composer. In this case, we are talking about what's known as algorithmic music; this area belongs to the Areopagus of computer music.

Creativity in the field of computer music requires certain types of computers, technical skills and specific knowledge. Thus, the computer algorithm could be constructed as a kind of "unchangeable" construction, the control of which is limited (e.g. limited by a pitch level, playback speed and ability of "rearrangement" of the elements performed). This construction, in spite of its apparent complexity, is only limited scope for the creative imagination of the composer.

More complicated is the algorithm allowing the ability of change from the part of the musician (system programmer or user). In this case, the algorithmic computer interactive music appears on the stage. It, in turn, may follow some theoretical probability models.

However, another process, being rather rigidly deterministic, can be laid in the basis of the machine algorithm. This process determines generation and evolution of the author's mathematical music. The author developed a musical and acoustic MARC model, one of the objectives of which is to study interaction between radiation and material. The model describes the evolution of rare planetary atmospheres under the influence of long-wave radiation (upper stratosphere of Earth and in the atmosphere of Mars).

The author's algorithm for constructing acoustic sonorities is based on the formation of the elements of the set of numbers as numerical experiment based on MARC model. In compliance with these elements, the acoustic frequencies 
are set. In the simplest case, the pace, timbre and amplitude of thus obtained computer music signal are fixed in advance and are not changeable over time. Thus, only the acoustic frequency changes; but just the variability of frequency generates the monothematic electronic melody.

The use of the programs - music editors provides a great opportunity for musical-acoustic experiments. Thus, the monothematic signal in MIDI format is easily divided into several sections, placed on different tracks, which leads to many-voiced (polyphonic) sound, as well as to variations in timbre and amplitude. The transformation to the wave format provides access to an extensive palette of "processing" and "effects" contained in the music editor menu. The use of one (or more) processes and/or effects allows, in addition to amplitude variations, to make significant changes in timbre. For example, the inclusion of a multiple reflection effect makes it possible to form the entire "line" of the so-called resonant sounding: from a vibrantly decorated harmony to a developed sonority, under which only sound registers (instead of single notes) are identifiable.

The author's musical and statistical MQS model allows analyzing specifics of the sound obtained on the basis of certain principles of nonlinear dynamics and mathematical statistics (reconstruction of images, calculation of correlation dimension, estimation of quantization noises, etc.) these features are not available to normal, "traditional" musical analysis.

Thus, we have a technological line, which lies in the generation of numerical sets, conversion of numbers into acoustic frequencies and computer analysis of sounding. This technological line is considered by us as computer and music simulation [6]. Please note that none of the elements of musical sounding is created "by hand": all musical examples are purely of computer origin.

Initial numeric arrays, as discussed above, are formed as a result of computer experiments. The musical acoustic model is based on a system of three (now-six) partial differential equations related to the so-called parabolic type. This type of equations describes the diffusion processes; the introduction of variable turbulent exchange coefficients converts the original equations into the class of quasi-linear ones. However, the consideration of absorption and emission processes described essentially by nonlinear (exponential) functions, complicates the problem even more. In reality, we deal with a system of three (or six) nonlinear equations of right-hand sides; computer modeling is virtually the only way to study the properties of these formalisms.

The significance of mathematical music from the standpoint of computer modeling of musical thinking is that the whole process of obtaining computer sonorities seems absolutely known to us-from composition of initial program to obtaining and processing audio material. Thus, the creative process can be represented in a simplified form as a certain algorithm implemented. 3".

One sample of mathematical music is represented "Fig.

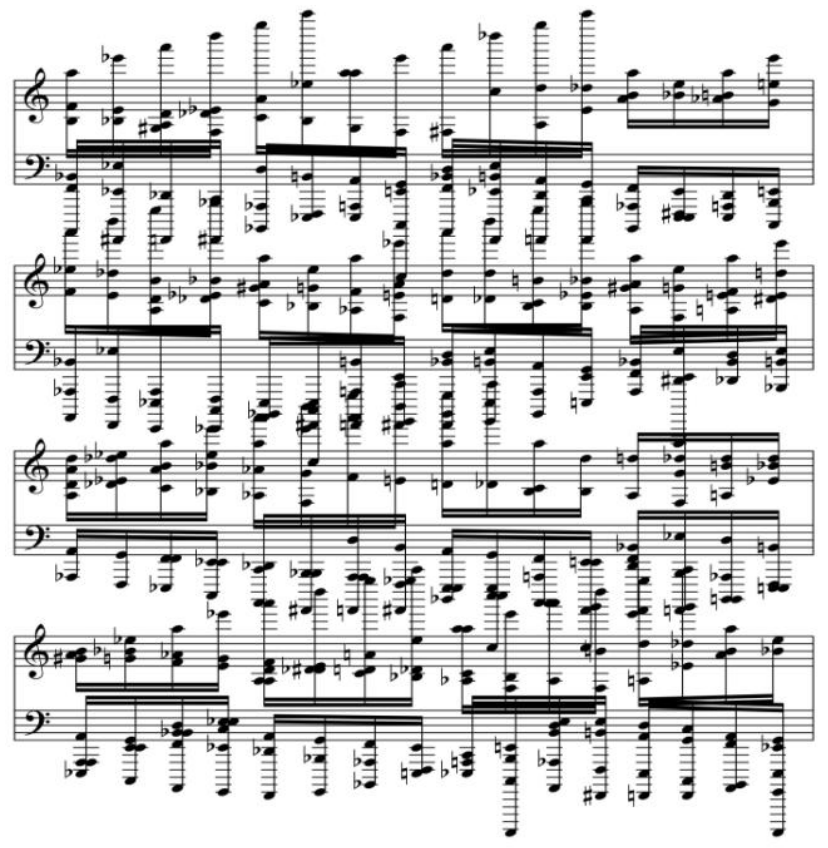

Fig. 3. Sample of mathematical music, 13 voices

\section{CONCLUSION}

It is believed that the phase reconstruction is one of the possible options for graphical imaging of system response. Thus, it is necessary to account for the fact that the response is currently deemed quite simplistic. However, further development of computer analysis methods of the properties of music signals on the basis of the author's musical and statistical model [6] allows us to introduce a number of additional variables (information entropy, fractal characteristics, etc.). The computer research of music signals produces a perspective in the study of the musical thinking modeling that means moving along the path predetermined by S. Skrebkov.

\section{REFERENCES}

[1] A.N. Kolmogorov, S.V. Fomin, Elements of the theory of functions and functional analysis. Second edition, revised and enlarged. M.,"Nauka ", 1968. - 496 pp., illustrated.

[2] A. Lebesgue. Integration and finding primitive functions. M.: GTTI 1934 .

[3] G.G. Malinetsky, A.B. Potapov. Modern problems of nonlinear dynamics. - M.: Editorial URSS , 2000. - 336 pp.

[4] A.B. Sergienko. Digital Signal Processing: Textbook for high schools. Second edition. - SPb.: Peter, 2007. - 751 pp.: illustrated.

[5] Sergey S. Skrebkov. Musician. Scientist. Teacher. Thinker (To the 100th anniversary) / Editor: D.A. Arutyunov, M.S. SkrebkovaFilatova, S.A. Filatov-Beckmann. - M.: Publishing House of NPF "TS-PRIMA"; Moscow State Tchaikovsky Conservatory. 2005. - 290 pp.: 11 lists of illustrations, portraits.

[6] S.A. Filatov-Beckmann. Computer-musical modeling: Textbook for high schools. - M.: "Sam Polygraphist" LLC, 2015. - 160 pp.: illustrated, music texts.

[7] Penrose R. Shadows of the Mind. Vintage, 1995. 